\title{
Improved Visualization of Cortical Lesions in Multiple Sclerosis Using 7T MP2RAGE
}

\author{
(DE.S. Beck, (D) P. Sati, DV. Sethi, (D)T. Kober, (D) B. Dewey, DP. Bhargava, (D) G. Nair, (DI.C. Cortese, and (DD.S. Reich
}

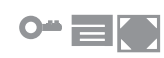

\begin{abstract}
BACKGROUND AND PURPOSE: Cortical lesions are common and often extensive in multiple sclerosis but are difficult to visualize by MRI, leaving important questions about their clinical implications and response to therapy unanswered. Our aim was to determine whether cortical lesions are better visualized using magnetization prepared 2 rapid acquisition gradient echoes (MP2RAGE) than T2*-weighted imaging on 7T MR imaging.
\end{abstract}

MATERIALS AND METHODS: Brain MR imaging using T1-weighted MP2RAGE at 500- $\mu \mathrm{m}$ isotropic resolution, T2*-weighted gradient-echo, and T2*-weighted segmented echo-planar imaging sequences were collected for 13 patients with MS and 5 age-matched neurologically healthy controls on a 7T research system. One MS case underwent postmortem MR imaging including gradient-echo and MP2RAGE sequences, after which cortical lesions seen on MR imaging were assessed with immunohistochemistry.

RESULTS: MP2RAGE detected 203 cortical lesions (median, 16 lesions/case; interquartile range, 15), compared to 92 with T2* gradient-echo (median, 7; interquartile range, $8 ; P<.001$ ) and 81 with T2*EPI (median, 7; interquartile range, 5; $P<.001$ ). This increase in lesion number detected on MP2RAGE versus T2* was observed for juxtacortical, leukocortical, and intracortical lesions. Forty-three percent of all cortical lesions were identified only on MP2RAGE. White matter lesion volume correlated with total juxtacortical $(r=0.86, P<.001)$ and leukocortical lesion volume $(r=0.70, P<.01)$ but not intracortical lesion volume, suggesting that pathophysiology may differ by lesion type. Of 4 suspected lesions seen on postmortem imaging, 3 were found to be true cortical lesions while 1 represented postmortem tissue damage.

CONCLUSIONS: A combination of MP2RAGE and T2*-weighted imaging at 7T improved detection of cortical lesions and should enable longitudinal studies to elucidate their spatiotemporal dynamics and clinical implications.

ABBREVIATIONS: GRE = gradient recalled-echo; $I Q R=$ interquartile range; MP2RAGE = magnetization prepared 2 rapid acquisition gradient echoes

M $\mathrm{S}$ is a demyelinating disorder affecting both white and gray matter. ${ }^{1-3}$ In some individuals, cortical demyelination exceeds white matter demyelination, ${ }^{2}$ and cortical lesions may be more strongly associated with disability and disability progression than white matter lesions. ${ }^{4-8}$ Cortical and white matter lesions

Received August 10, 2017; accepted after revision November 15.

From the Translational Neuroradiology Section (E.S.B., P.S., V.S., B.D., G.N., D.S.R.) and Neuroimmunology Clinic (I.C.C.), National Institute of Neurological Disorders and Stroke, National Institutes of Health, Bethesda, Maryland; Advanced Clinical Imaging Technology Group (T.K.), Siemens Healthcare Switzerland, Lausanne, Switzerland; Department of Radiology (T.K.), Centre Hospitalier Universitaire Vaudois, Lausanne, Switzerland; LTS5, Ecole Polytechnique Fédérale de Lausanne (T.K.), Lausanne, Switzerland; and Department of Neurology (P.B.), Johns Hopkins University, Baltimore, Maryland.

This work was supported by the Intramural Research Program of the National Institute of Neurological Disorders and Stroke, National Institutes of Health. E.S.B. is further supported by a Clinician Scientist Development Award from the National Multiple Sclerosis Society. P.B. is supported by a career transition award from the National Multiple Sclerosis Society, a Young Investigator Award from Race to Erase MS, and a John F. Kurtzke Clinician Scientist Development Award from the American Academy of Neurology. have potentially distinct origins and levels of inflammation, ${ }^{3,9-13}$ suggesting that they may have different clinical significance and response to treatment. Finally, the clinical course of MS is often not fully explained by volume and accumulation of white matter lesions, ${ }^{14}$ making better understanding of other types of MS pathology, including cortical lesions, imperative.

Although MR imaging is essential for MS diagnosis and disease monitoring, our limited understanding of cortical lesions stems from difficulties in imaging these lesions due to their size, myelin content, level of inflammation, and partial volume aver-

Please address correspondence to Daniel S. Reich, MD, PhD, Translational Neuroradiology Section, National Institute of Neurological Disorders and Stroke, National Institutes of Health, Building 10, Room 5C103, 10 Center Drive, Bethesda, MD 20892; e-mail: reichds@ninds.nih.gov

- Indicates open access to non-subscribers at www.ajnr.org

EIndicates article with supplemental on-line appendix and tables.

Indicates article with supplemental on-line photos.

http://dx.doi.org/10.3174/ajnr.A5534 
Table 1: MRI acquisition parameters for the 3 sequences acquired at 7T

\begin{tabular}{lccc} 
& 3D-MP2RAGE & 2D-T2*GRE & 3D-Segmented T2*EPI \\
\hline Orientation & Axial & Axial & Axial \\
Voxel dimensions $(\mu \mathrm{m})$ & $500 \times 500$ & $215 \times 215$ & $500 \times 500$ \\
Slice thickness $(\mu \mathrm{m})$ & 500 & 1000 & 500 \\
FOV $(\mathrm{mm})$ & $224(\mathrm{AP}) \times 168(\mathrm{RL}) \times 112(\mathrm{SI})$ & $220(\mathrm{AP}) \times 165(\mathrm{RL}) \times 25(\mathrm{SI})$ & $220(\mathrm{AP}) \times 180(\mathrm{RL}) \times 88(\mathrm{SI})$ \\
TI $(\mathrm{ms})$ & $800 / 2700$ & $\mathrm{NA}$ & $\mathrm{NA}$ \\
TR $(\mathrm{ms})$ & 6000 & 1300 & 52 \\
TE $(\mathrm{ms})$ & 5 & 32 & 23 \\
Flip angle & $4^{\circ} / 5^{\circ}$ & $50^{\circ}$ & $10^{\circ}$ \\
EPI factor & $\mathrm{NA}$ & $\mathrm{NA}$ & 15 \\
Scan time (min:sec) & $10: 32$ & $8: 36$ & $3: 40$ \\
No. of repetitions & 4 & 1 & 1 \\
\hline
\end{tabular}

Note:-AP indicates anterior-posterior axis; RL, right-left axis; SI, superior-inferior axis; NA, not applicable.

aging with CSF. Double inversion recovery and phase-sensitive inversion recovery sequences at $1.5 \mathrm{~T}$ and $3 \mathrm{~T}$ and $\mathrm{T} 2{ }^{\star}$-weighted sequences at 7T have been used to visualize cortical lesions. ${ }^{15-18}$ 7T MR imaging allows much higher spatial resolution, which is particularly important for the visualization of small cortical lesions, and $\mathrm{T} 2^{\star}$-weighted sequences at $7 \mathrm{~T}$ have been shown to more sensitively and reliably detect cortical lesions than double inversion recovery at $3 \mathrm{~T} .{ }^{19}$ However, small intracortical lesions remain difficult to visualize using all 3 of these techniques, and overall sensitivity remains low. ${ }^{20-22}$

Here, we aimed to develop an approach that could be used to investigate the natural history of cortical lesions in future studies, rather than develop an imaging sequence for routine clinical use. Recent studies in humans and nonhuman primates have demonstrated the value of T1-based MR imaging for visualizing the myeloarchitecture of the cortex. ${ }^{23-25}$ Among the different T1 MR imaging techniques, magnetization prepared 2 rapid acquisition gradient echoes (MP2RAGE) provides the best tissue contrast at high spatial resolution while minimizing field inhomogeneities that affect other techniques at $7 \mathrm{~T} .{ }^{26,27}$ We achieved high resolution while preserving image quality by combining multiple repetitions of the same 3D MP2RAGE acquisition. We also compared visualization of cortical lesions on MP2RAGE with 2 different T2* -weighted acquisitions.

\section{MATERIALS AND METHODS \\ In Vivo MR Imaging}

Thirteen participants in our MS natural history study and 5 neurologically healthy, age-matched controls (On-line Table 1) underwent brain MR imaging on a 7T whole-body research system (Siemens, Erlangen, Germany) equipped with a single-channel transmit, 32-channel phased array receive head coil. To maximize cortical lesion count, we included patients with MS with prior high-quality 7T scans and longstanding disease. The MP2RAGE sequence used previously optimized parameters ( $\mathrm{Ta}-$ ble 1$),{ }^{28}$ covered the entire cerebrum, was acquired at $500-\mu \mathrm{m}$ isotropic resolution, and was repeated 4 times to improve image quality (total scan time: $\sim 40$ minutes; On-line Fig 1 ). A $2 \mathrm{D}$ T2 ${ }^{*}$ gradient recalled-echo (GRE) sequence $(215 \times 215 \times 1000 \mu \mathrm{m})$, like one previously demonstrated to visualize cortical lesions, ${ }^{18}$ was acquired in 3 partially overlapping volumes to cover most of the cerebrum (total scan time, $\sim 25$ minutes). A novel 3D segmented T2 ${ }^{\star}$ EPI prototype sequence ${ }^{29,30}$ was acquired in 2 partially overlapping volumes at the same resolution as MP2RAGE (total scan time, $\sim 7$ minutes) (Table 1 ). In 2 patients with MS, the $\mathrm{T} 2{ }^{\star}$ sequences were acquired during the same session as MP2RAGE; in the other 11 , one or both $\mathrm{T} 2{ }^{\star}$ sequences were acquired on a separate day. The interval between MP2RAGE and GRE or EPI ranged from -8 to +18 months; in 10 of the patients with MS, MP2RAGE was acquired first. All patients with MS were also scanned on a 3T Magnetom Skyra scanner (Siemens) equipped with a 32-channel head coil (On-line Table 2).

\section{Image Processing}

Initial MP2RAGE processing to generate uniform images and T1 maps was performed on the scanner as part of the Siemens research sequence package (Work-in-Progress Package \#900B). ${ }^{26}$ The 4 repetitions were coregistered, and the voxelwise median was used for all analyses. T2* GRE and $\mathrm{T} 2{ }^{\star} \mathrm{EPI}$ were aligned to the MP2RAGE using linear coregistration. See the On-line Appendix for further details.

\section{Lesion Identification and Characterization}

Image analysis was conducted using a freely available image analysis software (Medical Image Processing, Analysis, and Visualization; National Institutes of Health; https://mipav.cit.nih.gov/). Images were magnified using bilinear interpolation as needed, and raters could manipulate window and level settings. Lesions were identified in the axial plane, with the option to confirm them on sagittal and coronal images. Lesions had to be hypointense on MP2RAGE images and/or hyperintense on $\mathrm{T} 2{ }^{\star}$ images and visible on at least 2 contiguous axial slices. Lesions were classified as intracortical (no white matter involvement; includes subpial lesions), leukocortical (involving both white matter and cortex), or juxtacortical (white matter lesions abutting but not clearly involving the cortex). Data were then compared across sequences. Comparison revealed that some cortical lesions were artifacts or white matter lesions that did not directly contact the cortex; these were excluded from further analyses.

E.S.B. (neurologist with 5 years' experience) marked cortical lesions on each sequence independently for all patients and controls, with at least 1 day between analyses of different sequences for the same individual. D.S.R. (neurologist/neuroradiologist with 14 years' experience) verified all lesions. Locations corresponding to lesions identified on only 1 or 2 sequences were examined on the remaining sequences to determine whether these lesions could be identified retrospectively. All lesions identified by E.S.B. and verified by D.S.R. (total 309) were included in lesion 
volume, count, and $\mathrm{T} 1$ relaxation time analyses. Lesions within the FOV for all 3 sequences (total 220) were used for sequencecomparison analyses. In 1 patient, $\mathrm{T} 2{ }^{\star} \mathrm{GRE}$ images were too motion-degraded to analyze, and lesions identified on MP2RAGE and $\mathrm{T} 2{ }^{*} \mathrm{EPI}$ for this patient were included in all analyses except for sequence comparisons.

To determine interrater agreement, P.B. (neurologist with 7 years' experience) marked cortical lesions on each sequence independently for 5 of the 13 patients with MS and all controls, with verification of all lesions by D.S.R. These ratings were then compared with those of E.S.B. for the same patients and controls. To determine intrarater agreement, E.S.B. re-analyzed the same 5 patients with MS several months after the first set of ratings.

To compute lesion volume, a single rater (E.S.B.) delineated ROIs manually, along the border of each lesion on all slices in which the lesion appeared on MP2RAGE. Total white matter lesion volume was also estimated using 3T FLAIR and MP2RAGE images. Images were registered to a brain atlas, ${ }^{31}$ and lesions were then segmented with LesionTOADS software (https://www. nitrc.org/projects/toads-cruise). ${ }^{32}$ Segmentation results were manually reviewed and corrected as necessary.

T1 relaxation times were obtained for each lesion using the median MP2RAGE T1 map. To obtain T1 values for normalappearing tissue and control tissue, we randomly chose 1 intracortical, 1 leukocortical, and 1 juxtacortical lesion for each case. For normal-appearing cortex and white matter, a contralateral ROI on a single slice was selected for each lesion. For control cortex and white matter, an ROI on a single slice matching each of the chosen lesions was selected in a control brain; ROIs were distributed evenly among the 5 control brains.

\section{Statistics}

The Kruskal-Wallis test with correction for multiple comparisons allowed cross-sequence comparison of lesion counts as well as lesion subtype count and volume analyses. One-way analysis of variance with correction for multiple comparisons allowed comparison of T1 relaxation times. Spearman correlation coefficients linked the volume of the cortical lesion subtypes with that of white matter lesions.

\section{Standard Protocol Approvals, Registrations, and Consents}

The study received approval from the NIH institutional review board, where the study was performed. All participants provided written informed consent. The next of kin for the person whose brain was evaluated postmortem provided written informed consent for the brain postmortem examination and use of material and clinical information for research purposes.

\section{RESULTS}

\section{More Cortical Lesions were Identified on MP2RAGE than on T2*-Weighted Images In Vivo at $7 T$}

$7 \mathrm{~T}$ images from 13 patients with MS and 5 neurologically healthy controls were analyzed (demographics in On-line Table 1). Three hundred nine cortical lesions were identified in the patients with MS (median, 24; range, 3-62; interquartile range [IQR], 15), including 85 juxtacortical (median, 5; range, 0-29; IQR, 5), 164 leukocortical (median, 14; range, 1-30; IQR, 12), and 60 intracortical (median, 3; range, 0-13; IQR, 3) lesions. Figure 1 shows representative examples of lesion appearance across sequences. Small intracortical lesions identified on MP2RAGE were often visible in retrospect on $\mathrm{T} 2{ }^{\star} \mathrm{GRE}$ and/or T2*EPI (Fig $1 G-I$ ); the same was true on MP2RAGE for several lesions initially identified only on $\mathrm{T} 2^{\star}$ (see below). Some lesions initially classified as white matter or juxtacortical on $\mathrm{T}^{*}$ were reclassified after comparison with MP2RAGE (Fig $1 P-R$ ), where the gray-white junction was better defined. Cortical involvement of some leukocortical lesions, in particular U-shaped lesions at the depths of the sulci, was more obvious on $\mathrm{T} 2{ }^{\star}$ than on MP2RAGE, where cortical (particularly subpial) involvement was sometimes subtler (Fig $1 J-L$ ).

Almost twice as many cortical lesions were detected on MP2RAGE as on T2* (Fig 2 and Table 2), independent of lesion subtype. A single cortical lesion was identified in 1 of the 5 healthy controls; this lesion was leukocortical and seen only on MP2RAGE (On-line Fig 2).

Forty-nine percent $(108 / 220)$ of cortical lesions were identified on both MP2RAGE and at least 1 of the T2* sequences, and $43 \%$ (95/220) were identified only on MP2RAGE. Only 8\% (17/ 220) were identified on at least 1 of the $\mathrm{T} 2^{*}$ sequences but not on MP2RAGE. These numbers were similar for juxtacortical and leukocortical lesions (Fig 2). For intracortical lesions, 39\% (17/44) were identified on both MP2RAGE and at least 1 T2* sequence, whereas $36 \%$ (16/44) were identified on MP2RAGE only and $25 \%$ $(11 / 44)$ were identified only on $\mathrm{T} 2{ }^{\star}$.

The number of total cortical lesions identified on the $2 \mathrm{~T} 2^{*}$ sequences did not differ, nor did the number of any of the 3 cortical lesion subtypes (Fig 2 and Table 2). Lesion subtype and size did not differ between lesions identified on T2*EPI but not GRE and lesions identified on T2 ${ }^{\star} \mathrm{GRE}$ but not EPI (On-line Table 4).

Lesions initially identified only on 1 or 2 sequences were evaluated retrospectively on the remaining sequences. Eighty-eight percent (84/95) of lesions identified prospectively on MP2RAGE only were seen in retrospect on at least 1 of the $\mathrm{T} 2^{\star}$ sequences. Forty-six of the 84 lesions were small or had only subtle signal change on $\mathrm{T} 2{ }^{*}$ images, likely explaining the lack of prospective identification, while 36 of the 84 lesions were visible but did not have clear cortical involvement on the T2* images; only 2 of the 84 lesions had obvious cortical involvement on $\mathrm{T} 2 *$ images, which had been missed in the initial analysis. All 17 lesions prospectively identified on $\mathrm{T}^{*}$ sequences but not on MP2RAGE were seen retrospectively on MP2RAGE images. Eleven of the 17 lesions were small or had only subtle signal changes on MP2RAGE, 2 lesions did not appear to involve the cortex on MP2RAGE, and 4 lesions had obvious cortical involvement on MP2RAGE but had been missed in the initial analysis. Ninety-eight percent $(43 / 44)$ of the lesions identified on $\mathrm{T} 2{ }^{*} \mathrm{GRE}$ but not $\mathrm{T} 2{ }^{\star} \mathrm{EPI}$ were visible in retrospect on $\mathrm{T} 2{ }^{\star} \mathrm{EPI}$, and $94 \%$ (31/33) of the lesions identified on $\mathrm{T} 2{ }^{\star} \mathrm{EPI}$ but not $\mathrm{T} 2{ }^{\star} \mathrm{GRE}$ were visible in retrospect on $\mathrm{T} 2{ }^{\star} \mathrm{GRE}$.

\section{Inter- and Intrarater Variability is Higher for T2* than for MP2RAGE}

For the 5 patients with MS in whom cortical lesions were identified on all 3 sequences by 2 raters, $44 \%$ of verified lesions were 
MP2RAGE
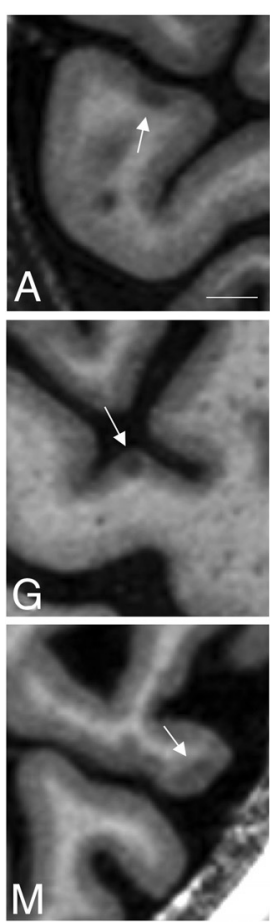

T2*GRE
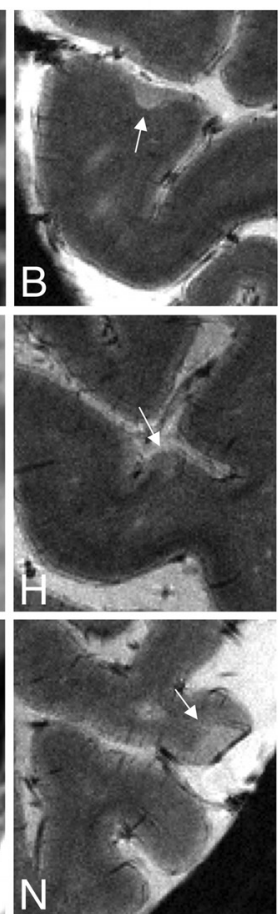

$\mathrm{T} 2{ }^{\star} \mathrm{EPI}$
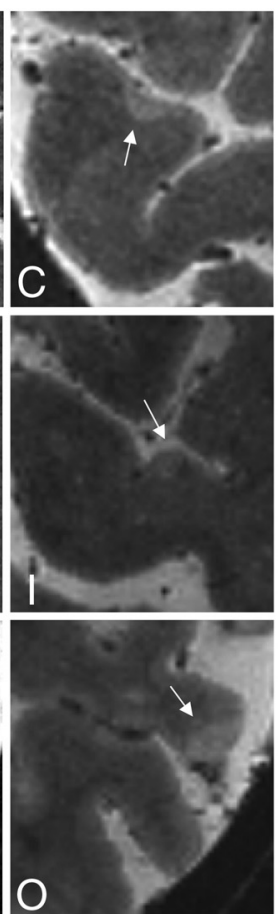

MP2RAGE
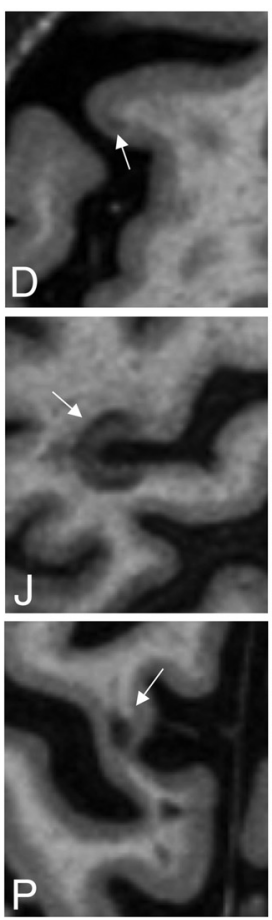

T2* GRE
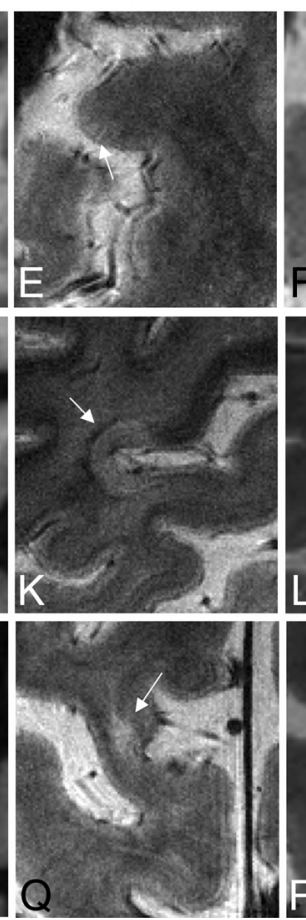

T2*EPI
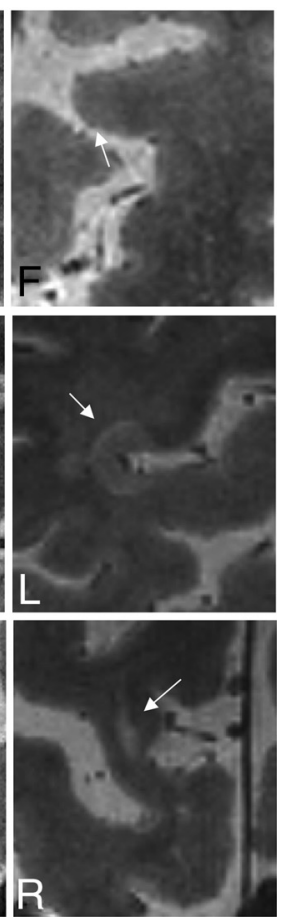

FIG 1. Comparison of cortical lesion detection with MP2RAGE and T2* weighted sequences at 7T. Intracortical (A-I), leukocortical (U-O), and juxtacortical $(P-R)$ lesions (arrows) are seen to different degrees on T1 MP2RAGE $(A, D, G, J, M$, and $P), T 2 * G R E(B, E, H, K, N$, and $Q)$, and T2*EPI $(C$, $F, I, L, O$, and $R$ ) sequences. $A-C$, An example of an intracortical lesion identified prospectively on all 3 sequences. $D-F$, An example of an intracortical lesion identified prospectively only on MP2RAGE and not visible even in retrospect on T2*GRE or T2*EPI. G-I, An example of an intracortical lesion identified prospectively only on MP2RAGE but visible in retrospect on T2*GRE and T2*EPI. J-L, An example of a leukocortical lesion, spanning the full cortical thickness and identified on all 3 sequences. $M-O$, An example of a leukocortical lesion, spanning the full cortical thickness and identified on T2*GRE and T2*EPI prospectively but only in retrospect on MP2RAGE. $P-R$, An example of a juxtacortical lesion identified as juxtacortical on MP2RAGE but initially incorrectly classified as a white matter lesion on GRE and EPI. Scale bar $=5 \mathrm{~mm}$.

identified on at least 1 sequence by both raters, whereas $56 \%$ of lesions were identified by only 1 rater. This discrepancy was greatest for intracortical lesions, of which only $26 \%$ were identified by both raters, compared with 51\% for leukocortical and 52\% for juxtacortical lesions. On MP2RAGE, $48 \%$ of lesions were identified by both raters; more than twice as many as were identified by both raters on $\mathrm{T} 2{ }^{\star} \mathrm{GRE}(23 \%)$ or T2*EPI $(22 \%)$.

Intrarater variability for the same 5 cases was similar to interrater variability, with $47 \%$ of lesions identified on at least 1 sequence by both raters and $53 \%$ of lesions identified only once. Fiftytwo percent of lesions identified on MP2RAGE were identified both times, compared with $40 \%$ on T2 ${ }^{\star} \mathrm{GRE}$ and $24 \%$ on T2 ${ }^{\star} \mathrm{EPI}$.

\section{Number and Volume of Cortical Lesion Subtypes Vary Widely across Patients}

Across the 13 patients with MS, there were more leukocortical (164) than intracortical (60) or juxtacortical (85) lesions. For each individual, however, the relative number of lesions of each type was highly variable, as was cortical lesion volume (On-line Fig 3 and On-line Table 5). Total white matter lesion volume did not correlate with intracortical lesion volume $(P=.44)$, but leukocortical lesion volume $(r=0.71, P=.009)$ and juxtacortical lesion volume $(r=0.86, P<.001)$ each correlated with total white matter lesion volume (Fig $3 A-C$ ).

When stratified by volume, $72 \%$ of the largest third of lesions (range, 55-2854 $\mu \mathrm{L}$; mean, $200 \mu \mathrm{L}$ ) were identified on MP2RAGE and at least $1 \mathrm{~T} 2^{\star}$ sequence, whereas $26 \%$ were identified only on MP2RAGE and 3\% were identified only on T2*. In contrast, for lesions with volumes in the middle third (range, $26-55 \mu \mathrm{L}$; mean, 40 $\mu \mathrm{L}), 46 \%$ were identified on both sequence types, whereas $41 \%$ were identified on MP2RAGE only and $14 \%$ on T2* only. For the smallest third of lesions (range, 5-26 $\mu \mathrm{L}$; mean, $15 \mu \mathrm{L}$ ), 30\% were identified on both sequence types; $63 \%$, on MP2RAGE only; and 7\%, on T2* only. Thus, larger lesions are more likely to be identified on both MP2RAGE and T2* sequences, whereas smaller lesions are more likely to be identified on only MP2RAGE.

\section{T1 Relaxation Times Differ across Cortical Lesion Types}

The mean T1 relaxation times were calculated for each cortical lesion, for normal-appearing gray and white matter in patients with MS, and for control cortex and white matter (Fig 3D). The mean T1 was higher in juxtacortical $(2062 \pm 230 \mathrm{~ms})$ than in leukocortical $(1961 \pm 182 \mathrm{~ms}, P<.001)$ or intracortical $(1871 \pm$ $96 \mathrm{~ms}, P<.001)$ lesions. Leukocortical lesions also had higher T1 than intracortical lesions $(P<.001)$. The mean $\mathrm{T} 1$ relaxation time for each lesion type was higher $(P<.001)$ than for the corresponding normal-appearing and control tissue. There was no difference between normal-appearing $(1703 \pm 90 \mathrm{~ms})$ and control $(1685 \pm 88 \mathrm{~ms})$ gray matter or between normal-appearing $(1261 \pm 92 \mathrm{~ms})$ and control $(1207 \pm 86 \mathrm{~ms})$ white matter. T1 of lesions identified on both MP2RAGE and at least $1 \mathrm{~T} 2 *$ sequence 
$(2012 \pm 213 \mathrm{~ms})$ was higher than that of lesions identified only on MP2RAGE $(1923 \pm 182 \mathrm{~ms}, P<.01)$ and those identified only on $\mathrm{T}^{*}$ sequences $(1893 \pm 87 \mathrm{~ms}, P<.05)$. The T1 of lesions identified on MP2RAGE only was not significantly different from that of lesions identified on $\mathrm{T}^{\star}$ only.

\section{Most Cortical Lesions Visualized by MP2RAGE on Postmortem 7T MR Imaging Can Be Verified Pathologically}

One postmortem MS brain was imaged at 7T with MP2RAGE and $\mathrm{T} 2{ }^{*} \mathrm{GRE}$ (On-line Appendix). The areas of tissue corresponding to 4 suspected leukocortical lesions, all with predominantly cortical involvement, were isolated and stained for myelin proteolipid protein. Three of the suspected lesions, all of which were identified prospectively on MP2RAGE and T2* GRE, were clearly demyelinated lesions (On-line Fig 4). The fourth, which was identified on $\mathrm{T} 2{ }^{*} \mathrm{GRE}$ prospectively and only seen in retrospect on MP2RAGE, was a small tear in the cortex that likely occurred during postmortem handling.

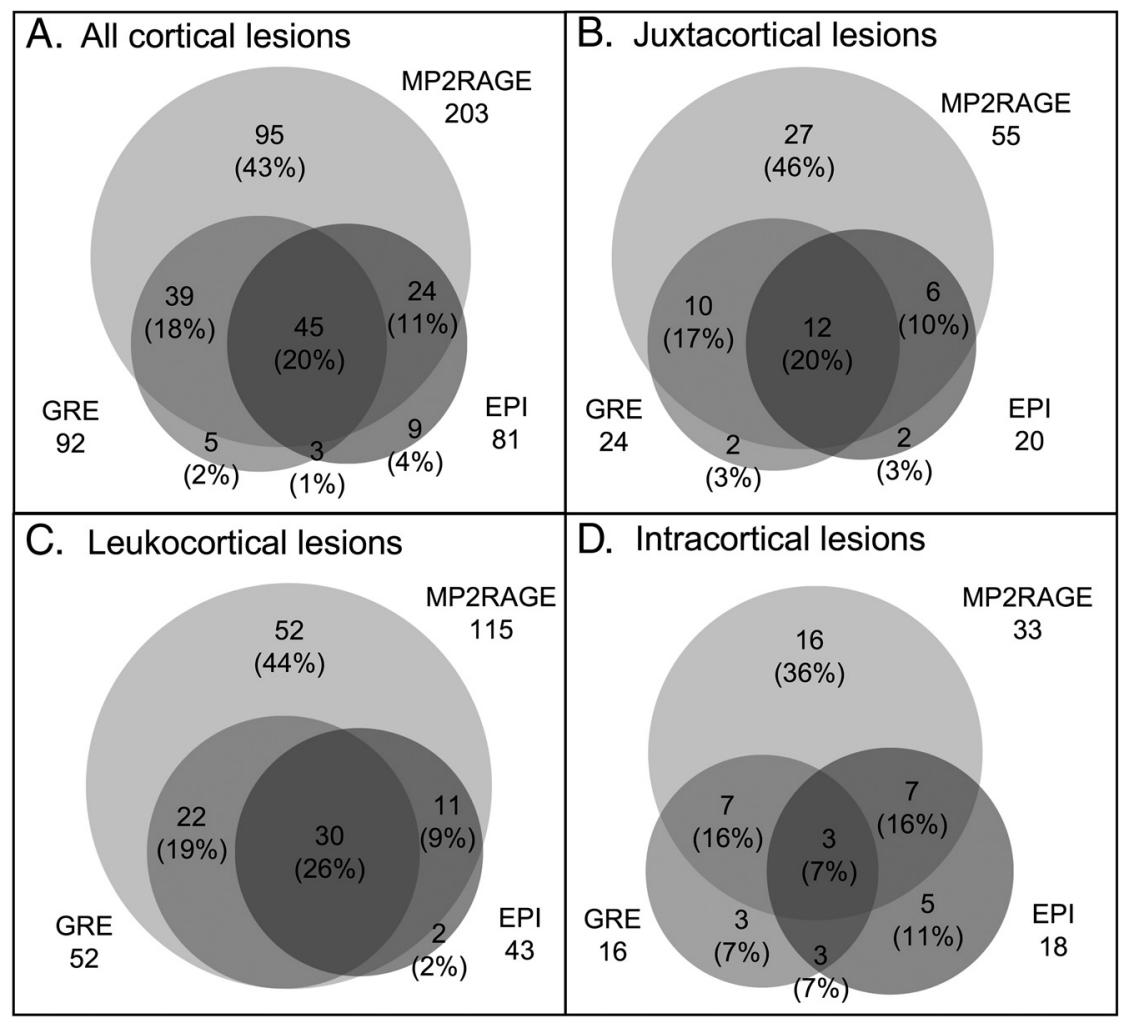

FIG 2. Increased cortical lesion detection with MP2RAGE. Venn diagrams show comparison of prospective lesion identification with MP2RAGE, T2* GRE, and T2*EPI for all cortical lesions and for each cortical lesion subtype.

\section{DISCUSSION}

In this study, we show that acquisition of high-resolution MP2RAGE at 7T dramatically increases the detection of cortical lesions compared with state-of-the-art $\mathrm{T} 2{ }^{\star} \mathrm{GRE}$ or our prototype T2*EPI sequence. Reasons for the superior sensitivity of MP2RAGE over T2* include more homogeneous signal intensity, better gray-white junction delineation, and decreased image artifacts. Importantly, we found that MP2RAGE allowed identification of smaller and more subtle lesions while generally preserving visualization of lesions identifiable on $\mathrm{T} 2^{*}$.

Our comparison of MP2RAGE images acquired in patients with MS and controls, our postmortem verification of several cortical lesions seen on MP2RAGE, and our retrospective identification of most lesions seen on MP2RAGE on $\mathrm{T} 2 *$ images together suggest that most identified cortical lesions are true demyelinated lesions. Although we cannot rule out that some cortical lesions identified in MS are due to other types of tissue damage or artifacts (also the case for white matter lesions), the number of such lesions is likely low.

Despite the advantages of MP2RAGE, some cortical lesions were seen better on $\mathrm{T} 2 *$ images, whereas others appeared to have different degrees of cortical involvement on MP2RAGE versus T2*. Some cortical lesions appeared subpial on $\mathrm{T}^{*}$ but not on MP2RAGE (eg, Fig $1 M-O)$. One potential explanation is that there are pathologic differences in lesions close to the pial surface versus deeper in the cortex, leading to differences in visualization on MR imaging. $\mathrm{T}^{*}$ sequences may also be particularly sensitive to cortical pathology due to differences in magnetic susceptibility between cortical layers. A more definitive answer to this question will require detailed postmortem MR imaging-histopathologic correlation.

Although we hypothesize that the ability of MP2RAGE and T2* to detect cortical lesions differs in part due to specific pathologic features of different lesions, most lesions identified on 1 or 2 sequences were visible on the remainder of the sequences in retrospect. When evaluated retrospectively, many lesions missed initially on $\mathrm{T} 2{ }^{*}$ were small or had

Table 2. Comparison of lesion identification on MP2RAGE, T2* ${ }^{*} \mathrm{RE}$, and T2*EPI images

\begin{tabular}{|c|c|c|c|c|c|c|c|c|c|c|c|c|}
\hline & \multicolumn{3}{|c|}{ MP2RAGE } & \multicolumn{3}{|c|}{ T2* GRE } & \multicolumn{3}{|c|}{ T2* EPI } & \multicolumn{3}{|c|}{ Significance } \\
\hline & \multirow[b]{2}{*}{ Total } & \multicolumn{2}{|c|}{ Per Patient } & \multirow[b]{2}{*}{ Total } & \multicolumn{2}{|c|}{ Per Patient } & \multirow[b]{2}{*}{ Total } & \multicolumn{2}{|c|}{ Per Patient } & \multirow[b]{2}{*}{$\begin{array}{c}\text { MP2RAGE } \\
\text { vs GRE }\end{array}$} & \multirow[b]{2}{*}{$\begin{array}{l}\text { MP2RAGE } \\
\text { vs EPI }\end{array}$} & \multirow[b]{2}{*}{$\begin{array}{c}\text { GRE } \\
\text { vs EPI }\end{array}$} \\
\hline & & Median & $\begin{array}{c}\text { Range, } \\
\text { IQR }\end{array}$ & & Median & $\begin{array}{c}\text { Range, } \\
\text { IQR }\end{array}$ & & Median & $\begin{array}{c}\text { Range, } \\
\text { IQR }\end{array}$ & & & \\
\hline All & 203 & 16 & $3-35,15$ & 92 & 8 & $0-21,8$ & 81 & 7 & $0-15,5$ & 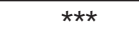 & 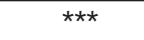 & NS \\
\hline Juxtacortical & 55 & 4 & $0-13,4$ & 24 & 2 & $0-18,2$ & 20 & 2 & $0-6,4$ & * & * & NS \\
\hline Leukocortical & 115 & 11 & $1-21,10$ & 52 & 4 & $0-11,4$ & 43 & 4 & $0-8,5$ & ** & $\star \star \star \star$ & NS \\
\hline Intracortical & 33 & 2 & $0-8,3$ & 16 & 1 & $0-5,2$ & 18 & 1 & $0-6,2$ & * & NS & NS \\
\hline
\end{tabular}

Note:-NS indicates not significant; asterisk, $P<.05$; double asterisks, $P<.01$; triple asterisks, $P<.001$ 


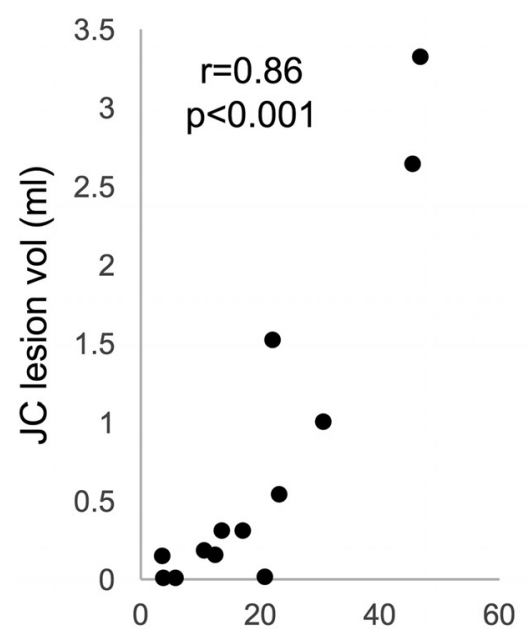

A White matter lesion vol (ml)

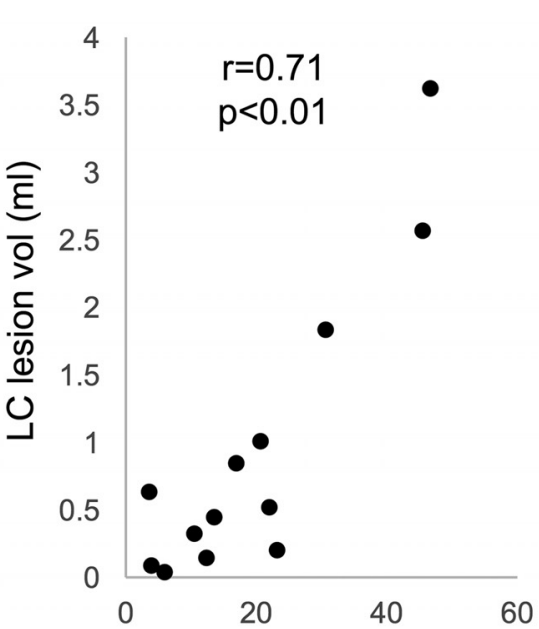

B White matter lesion vol (ml)

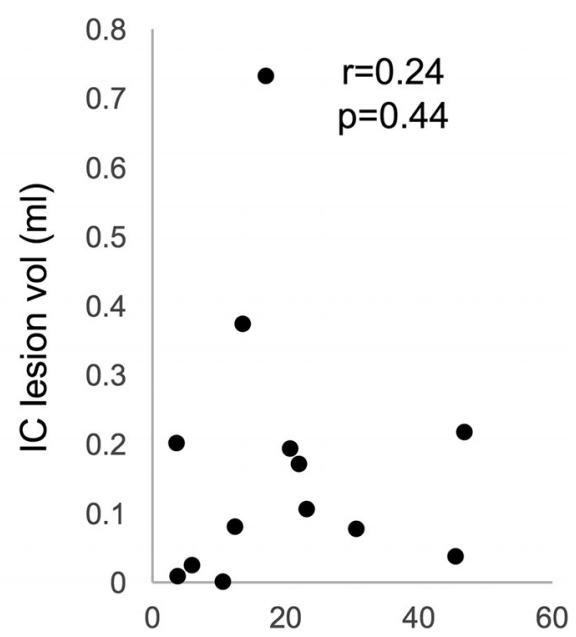

C White matter lesion vol $(\mathrm{ml})$

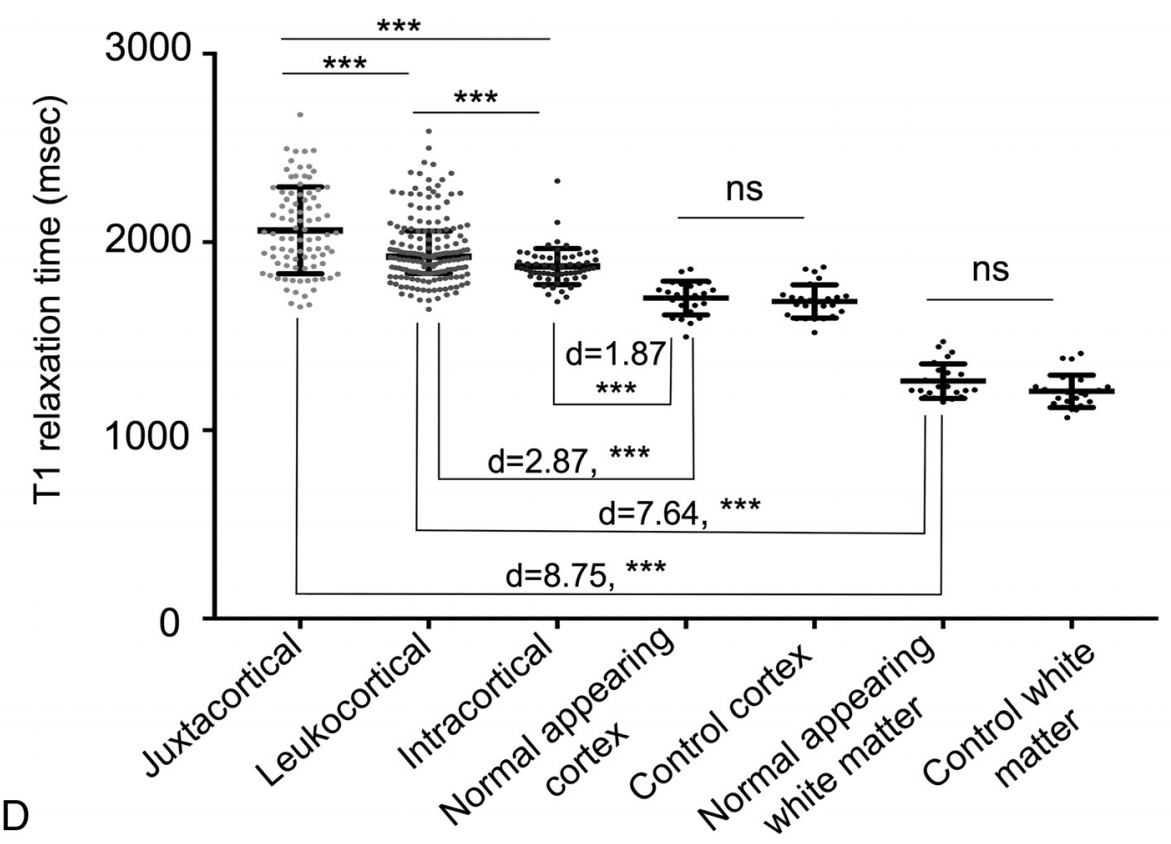

FIG 3. Cortical lesion volume and $T 7$ relaxation times. $A-C$, Total white matter lesion volume correlates with total juxtacortical lesion volume and leukocortical lesion volume but not with intracortical lesion volume. $D, T 1$ relaxation time is highest for juxtacortical lesions, followed by leukocortical and intracortical lesions. The $\mathrm{T} 7$ for all cortical lesion types exceeds that of normal-appearing and control tissues, though this difference is more dramatic for juxtacortical and leukocortical lesions compared with normal-appearing white matter versus leukocortical and intracortical lesions compared with normal-appearing gray matter. Normal-appearing tissue and control tissue did not differ in $\mathrm{Tl}$ value. $\mathrm{d}$ indicates effect size. Error bars represent SD. Three asterisks indicate, $P<.001$.

only a subtle signal change, which was compounded by the higher prevalence of motion artifacts on $\mathrm{T} 2^{\star}$ images and the increased slice thickness on $\mathrm{T} 2{ }^{\star} \mathrm{GRE}$. In addition, likely due to their small size and sometimes subtle nature, many cortical lesions are missed when a single rater evaluates an image, a particular problem with $\mathrm{T} 2{ }^{*}$. Thus, going forward, multiple raters, each using MP2RAGE and $\mathrm{T} 2 *$ images, will likely allow the most sensitive and accurate detection of cortical lesions. A computational method for identifying potential cortical lesions on the basis of T1 values that differ from those of the surrounding cortex may also be useful in the future for more comprehensive lesion detection.

One limitation to the sequence comparison performed here is that the MP2RAGE images used were derived from averaging 4 acquisitions and had a longer total acquisition time than the T2* sequences. Our goal was to identify as many cortical lesions as possible using sequences with optimal image quality; while this goal required averaging for MP2RAGE to achieve image quality similar to that of single acquisitions for the $\mathrm{T} 2{ }^{*}$ sequences, we believe that the comparison of sequences performed here is valid.

In this study, we subcategorized cortical lesions as intracortical, leukocortical, and juxtacortical but did not further subclassify intracortical lesions; even at high resolution, it was often difficult to distinguish intracortical lesions elsewhere categorized as types II, III, and IV. ${ }^{33}$ We also chose not to distinguish between leukocortical lesions that mostly affect the cortex versus those that mostly affect white matter because relative cortical and white matter involvement appears to be a continuum; however, it will be 
interesting to study cortical lesion evolution longitudinally to distinguish lesions that originate in the cortex versus white matter. The T2*EPI sequence appeared to be as good as T2 ${ }^{\star}$ GRE for the identification of cortical lesions, despite the larger voxel volume and substantially shorter imaging time. We therefore propose using T2*EPI as a second sequence for cortical lesion identification, to confirm lesions identified on MP2RAGE and assess their subpial involvement.

Previous work correlating different types and severity of MS with cortical lesion number has been, for the most part, performed at low field strength using double and phase-sensitive inversion recovery ${ }^{34-38}$; it is likely that cortical lesions identified in those studies were heavily skewed toward juxtacortical and leukocortical lesions, underestimating the number of intracortical lesions. ${ }^{39}$ With the increased sensitivity to small cortical lesions provided by MP2RAGE, we were able to more accurately determine the correlation between cortical and white matter lesions. We found that total juxtacortical and leukocortical but not intracortical lesion volume correlated with white matter lesion volume and that even this correlation may be driven by high-lesion-load cases, providing evidence that the pathophysiologic processes leading to cortical and white matter lesion formation and/or growth are distinct. Thus, it seems likely to us that following white matter lesion evolution may not yield a complete picture of the disease, and treatments that are effective in stopping new white matter lesions may not stop cortical lesion formation. We did not find any correlation between cortical lesion number or volume and clinical characteristics, but this may be due to the small sample size and our participant-selection method, which favored individuals with long-standing MS and known cortical lesions. Larger prospective studies using the methodology described here are therefore necessary.

MP2RAGE also provides T1 maps, which could further help quantitatively characterize lesions ${ }^{40}$ and tease out pathologic differences among lesion types. We show that T1 differs across cortical lesion subtypes. Differences between the T1 of lesions and their corresponding control tissue are smallest for intracortical lesions; these small differences make them harder to detect. Changes in cortical lesion T1 may prove useful as a marker for tissue destruction and recovery.

Although our combination of MR imaging sequences at 7T improves detection of cortical lesions, one major limitation is the long acquisition time required (approximately 70 minutes for all 3 sequences), restricting the use of this approach to the research setting. However, it may be possible to decrease the number of MP2RAGE scan repetitions for clinical use, decreasing sensitivity but still likely representing an improvement over the current standard. Future advances in phased array coil designs and parallel imaging methods may also allow reduction in scan time without substantial loss in lesion identification. Another limitation of our study is that MP2RAGE and T2* sequences were sometimes acquired several months apart, and we cannot fully discount the possibility that differences in lesion identification were due to lesion formation or evolution between MRIs. However, in almost all cases, T2* images were acquired after MP2RAGE images, so the increased lesion identification on MP2RAGE cannot be attributed to new lesion formation between scans.

\section{CONCLUSIONS}

We demonstrate here that improved cortical lesion detection can be achieved in vivo by using a unique combination of MR imaging sequences at 7T. This advance may allow assessment of whether cortical lesions account for some of the clinical heterogeneity in MS that is not explained by white matter lesion load and could help better predict the course of disease and guide treatment.

\section{ACKNOWLEDGMENTS}

We thank Martina Absinta, Matthew Schindler, Souheil Inati, and the staff of the National Institutes of Health functional MRI Facility for help with MR imaging acquisitions. We also thank Joan Ohayon, Frances Andrada, and the staff of the National Institute of Neurological Disorders and Stroke Neuroimmunology Clinic for care of and collection of clinical data from the study participants. The National Cancer Institute Laboratory of Pathology and Seung Kown Ha assisted in acquiring and processing the pathologic material. Gina Norato of the National Institute of Neurological Disorders and Stroke Clinical Trials Unit helped with statistical analyses.

Disclosures: Erin S. Beck—RELATED: Grant: National Multiple Sclerosis Society, Comments: I am the recipient of a Clinician Scientist Development Award from the National Multiple Sclerosis Society*. Tobias Kober-RELATED: Employment: Siemens, Switzerland. Daniel S. Reich—RELATED: Grant: National Multiple Sclerosis Society, Comments: Fellowship Grant to Erin S. Beck*; UNRELATED: Other: Vertex Pharmaceuticals, Myelin Repair Foundation, Comments: Funds for research were transferred to my institution as part of Cooperative Research and Development Agreements*. *Money paid to the institution.

\section{REFERENCES}

1. Brownell B, Hughes JT. The distribution of plaques in the cerebrum in multiple sclerosis. J Neurol Neurosurg Psychiatry 1962;25:315-20 CrossRef Medline

2. Bø L, Vedeler CA, Nyland HI, et al. Subpial demyelination in the cerebral cortex of multiple sclerosis patients. J Neuropathol Exp Neurol 2003;62:723-32 CrossRef Medline

3. Lucchinetti CF, Popescu BF, Bunyan RF, et al. Inflammatory cortical demyelination in early multiple sclerosis. N Engl J Med 2011;365: 2188-97 CrossRef Medline

4. Calabrese M, Agosta F, Rinaldi F, et al. Cortical lesions and atrophy associated with cognitive impairment in relapsing-remitting multiple sclerosis. Arch Neurol 2009;66:1144-50 Medline

5. Calabrese M, Poretto V, Favaretto A, et al. Cortical lesion load associates with progression of disability in multiple sclerosis. Brain 2012;135:2952-61 CrossRef Medline

6. Harrison DM, Roy S, Oh J, et al. Association of cortical lesion burden on 7-T magnetic resonance imaging with cognition and disability in multiple sclerosis. JAMA Neurol 2015;72:1004-12 CrossRef Medline

7. Kutzelnigg A, Lucchinetti CF, Stadelmann C, et al. Cortical demyelination and diffuse white matter injury in multiple sclerosis. Brain 2005;128:2705-12 CrossRef Medline

8. Calabrese M, Romualdi C, Poretto V, et al. The changing clinical course of multiple sclerosis: a matter of gray matter. Ann Neurol 2013;74:76-83 CrossRef Medline

9. Magliozzi R, Howell O, Vora A, et al. Meningeal B-cell follicles in secondary progressive multiple sclerosis associate with early onset of disease and severe cortical pathology. Brain 2007;130:1089-104 Medline

10. Magliozzi R, Howell OW, Reeves C, et al. A gradient of neuronal loss and meningeal inflammation in multiple sclerosis. Ann Neurol 2010;68:477-93 CrossRef Medline

11. Howell OW, Reeves CA, Nicholas R, et al. Meningeal inflammation 
is widespread and linked to cortical pathology in multiple sclerosis. Brain 2011;134:2755-71 CrossRef Medline

12. Choi SR, Howell OW, Carassiti D, et al. Meningeal inflammation plays a role in the pathology of primary progressive multiple sclerosis. Brain 2012;135:2925-37 CrossRef Medline

13. Absinta M, Vuolo L, Rao A, et al. Gadolinium-based MRI characterization of leptomeningeal inflammation in multiple sclerosis. Neurology 2015;85:18-28 CrossRef Medline

14. Filippi M, Charil A, Rovaris M, et al. Insights from magnetic resonance imaging. Handb Clin Neurol 2014;122:115-49 CrossRef Medline

15. Geurts JJ, Pouwels PJ, Uitdehaag BM, et al. Intracortical lesions in multiple sclerosis: improved detection with 3D double inversion-recovery MR imaging. Radiology 2005;236:254-60 CrossRef Medline

16. Calabrese M, De Stefano N, Atzori M, et al. Detection of cortical inflammatory lesions by double inversion recovery magnetic resonance imaging in patients with multiple sclerosis. Arch Neurol 2007; 64:1416-22 CrossRef Medline

17. Nelson F, Poonawalla AH, Hou P, et al. Improved identification of intracortical lesions in multiple sclerosis with phase-sensitive inversion recovery in combination with fast double inversion recovery MR imaging. AJNR Am J Neuroradiol 2007;28:1645-49 CrossRef Medline

18. Mainero $\mathrm{C}$, Benner $\mathrm{T}$, Radding $\mathrm{A}$, et al. In vivo imaging of cortical pathology in multiple sclerosis using ultra-high field MRI. Neurology 2009;73:941-48 CrossRef Medline

19. Nielsen AS, Kinkel RP, Tinelli E, et al. Focal cortical lesion detection in multiple sclerosis: 3 Tesla DIR versus 7 Tesla FLASH-T2. J Magn Reson Imaging 2012;35:537-42 CrossRef Medline

20. Geurts JJ, Bö L, Pouwels PJ, et al. Cortical lesions in multiple sclerosis: combined postmortem MR imaging and histopathology. AJNR Am J Neuroradiol 2005;26:572-77 Medline

21. Pitt D, Boster A, Pei W, et al. Imaging cortical lesions in multiple sclerosis with ultra-high-field magnetic resonance imaging. Arch Neurol 2010;67:812-18 CrossRef Medline

22. Jonkman LE, Klaver R, Fleysher L, et al. Ultra-high-field MRI visualization of cortical multiple sclerosis lesions with $\mathrm{T} 2$ and $\mathrm{T} 2{ }^{\star}$ : a postmortem MRI and histopathology study. AJNR Am J Neuroradiol 2015;36:2062-67 CrossRef Medline

23. Haast RA, Ivanov D, Formisano E, et al. Reproducibility and reliability of quantitative and weighted $\mathrm{T} 1$ and $\mathrm{T} 2$ * mapping for myelinbased cortical parcellation at 7 Tesla. Front Neuroanat 2016;10:112 CrossRef Medline

24. Geyer S, Weiss M, Reimann K, et al. Microstructural parcellation of the human cerebral cortex: from Brodmann's post-mortem map to in vivo mapping with high-field magnetic resonance imaging. Front Hum Neurosci 2011;5:19 CrossRef Medline

25. Bock NA, Hashim E, Kocharyan A, et al. Visualizing myeloarchitec- ture with magnetic resonance imaging in primates. Ann N Y Acad Sci 2011;1225(suppl 1):E171-81 CrossRef Medline

26. Marques JP, Kober T, Krueger G, et al. MP2RAGE, a self bias-field corrected sequence for improved segmentation and T1-mapping at high field. Neuroimage 2010;49:1271-81 CrossRef Medline

27. O'Brien KR, Kober T, Hagmann P, et al. Robust T1-weighted structural brain imaging and morphometry at 7T using MP2RAGE. PLoS One 2014;9:e99676 CrossRef Medline

28. Marques JP, Gruetter R. New developments and applications of the MP2RAGE sequence: focusing the contrast and high spatial resolution R1 mapping. PLoS One 2013;8:e69294 CrossRef Medline

29. Sati P, Thomasson DM, Li N, et al. Rapid, high-resolution, wholebrain, susceptibility-based MRI of multiple sclerosis. Mult Scler 2014;20:1464-70 CrossRef Medline

30. Sati P, Patil S, Inati S, et al. Rapid MR susceptibility imaging of the brain using segmented 3D echo-planar imaging (3D EPI) and its clinical applications. Magnetom FLASH 2017;68:26-32

31. Fonov V, Evans AC, Botteron K, et al; Brain Development Cooperative Group. Unbiased average age-appropriate atlases for pediatric studies. Neuroimage 2011;54:313-27 CrossRef Medline

32. Shiee N, Bazin PL, Ozturk A, et al. A topology-preserving approach to the segmentation of brain images with multiple sclerosis lesions. Neuroimage 2010;49:1524-35 CrossRef Medline

33. Peterson JW, Bö L, Mörk S, et al. Transected neurites, apoptotic neurons, and reduced inflammation in cortical multiple sclerosis lesions. Ann Neurol 2001;50:389-400 CrossRef Medline

34. Calabrese M, Filippi M, Rovaris M, et al. Evidence for relative cortical sparing in benign multiple sclerosis: a longitudinal magnetic resonance imaging study. Mult Scler 2009;15:36-41 Medline

35. Diker S, Has AC, Kurne A, et al. The association of cognitive impairment with gray matter atrophy and cortical lesion load in clinically isolated syndrome. Mult Scler Relat Disord 2016;10:14-21 CrossRef Medline

36. Absinta M, Rocca MA, Moiola L, et al. Cortical lesions in children with multiple sclerosis. Neurology 2011;76:910-13 CrossRef Medline

37. Papadopoulou A, Müller-Lenke N, Naegelin Y, et al. Contribution of cortical and white matter lesions to cognitive impairment in multiple sclerosis. Mult Scler 2013;19:1290-96 CrossRef Medline

38. Nelson F, Datta S, Garcia N, et al. Intracortical lesions by 3T magnetic resonance imaging and correlation with cognitive impairment in multiple sclerosis. Mult Scler 2011;17:1122-29 CrossRef Medline

39. Seewann A, Kooi EJ, Roosendaal SD, et al. Postmortem verification of MS cortical lesion detection with 3D DIR. Neurology 2012;78: 302-08 CrossRef Medline

40. Kober T, Granziera C, Ribes D, et al. MP2RAGE multiple sclerosis magnetic resonance imaging at $3 \mathrm{~T}$. Invest Radiol 2012;47:346-52 CrossRef Medline 